\title{
$P$ wie die Praxis in den Bergen oder wieso sie es trotzdem tun
}

\section{Dominik Heim}

PD Dr. med., Facharzt für Chirurgie

Nein, sagt er, er wisse beim besten Willen nicht mehr, wie das genau passiert sei, ob er wohl die Skis gekreuzt habe? Er weiss auch nicht mehr, dass man ihm vor Kurzem seine Stelle gekündigt habe, vielleicht sei es besser so, meint seine Begleiterin. Aber irgendwann wird auch diese Information wieder durchsickern, ob noch in der grossen Praxis, fast am Rand der Piste oder aber im nächsten Spital, das rund 50 Autominuten von hier im Tal unten liegt. Und ja, dieses ausgesprengte Fragment am Ellbogen, das sollte man wieder an den ursprünglichen Platz bringen, die Frage ist nur, ob man dies im Privatspital im Flachland oder im Spital da unten am Ende des Tals machen soll. Und dieses Ohrenweh beim 6-jährigen Kind ist beim Reinschauen ganz klar eine Mittelohrentzündung, einen Spezialisten zur Diagnostik braucht es nicht, die Frage ist höchstens, ob es wirklich Antibiotika dazu braucht.
Es gibt diese Praxen in den Alpen, die mit solchen und noch viel ganz anderen medizinischen Situationen konfrontiert sind, man muss auch katheterisieren, die Tonsillen beurteilen, die Pneumonie hören oder sehen und jemandem auch einmal sagen, dass es wirklich nichts sei, und dass auch der erwünschte Spezialist, mindestens der somatische, einfach nichts anderes machen könne. Es gibt dann auch Patienten, die glauben, dass ihr Geld oder ihr Auto sie zu einer besonderen Klasse von Patienten macht, und es gibt solche, wo es einem selbst leid tut, dass man der Überbringer von schlechten Nachrichten ist, ob es nun ein völlig zertrümmerter Schienbeinkopf ist oder ein maligner Lymphknoten, den man herausgenommen hat. Wie im Film «le médecin de campagne» [1] schon gesagt, spielt die Anamnese für die Diagnose eine eminent wichtige Rolle, und so wird auch hier gefragt

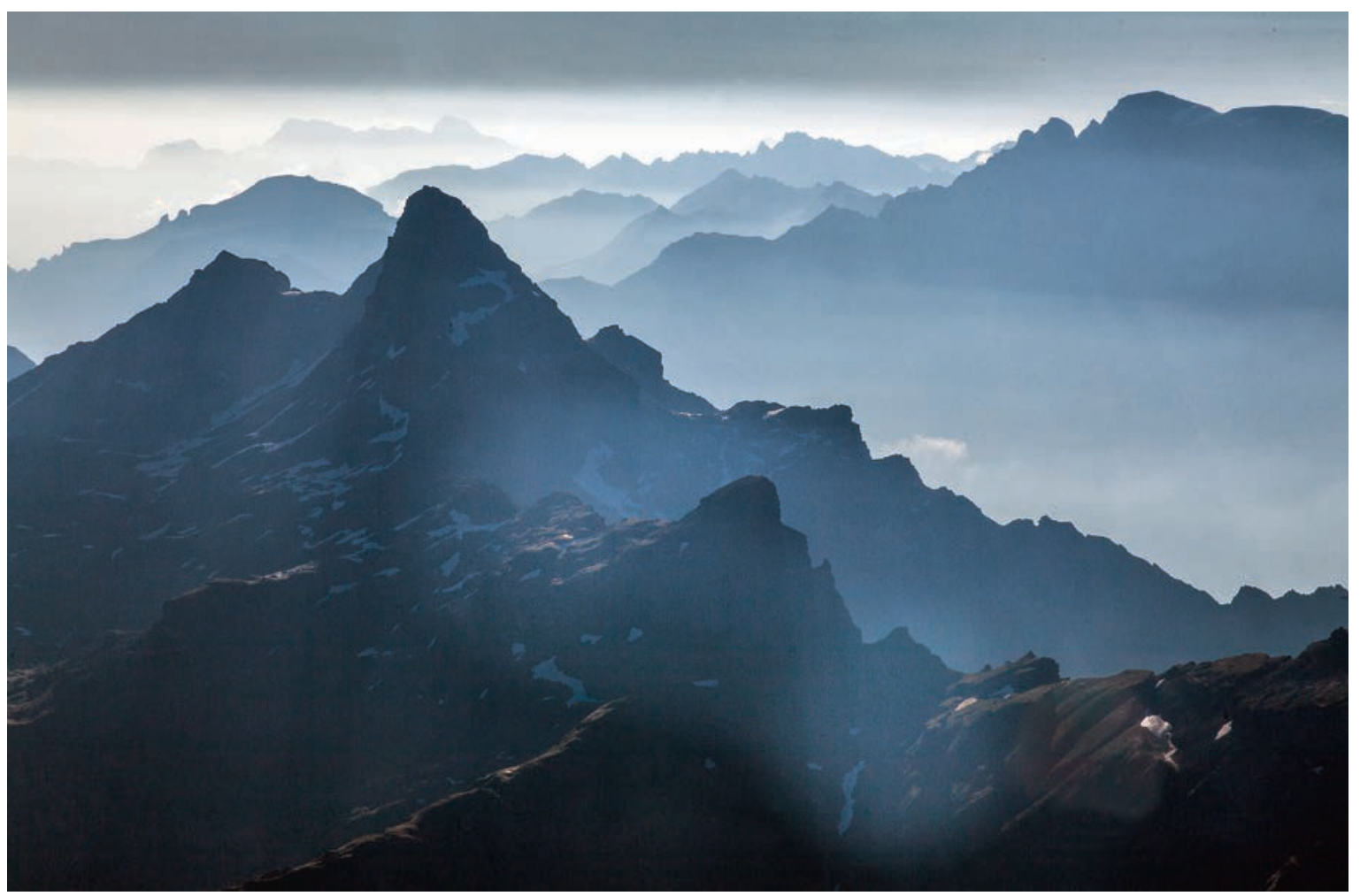

Der Weg ins Tal ist weit, es hat dort Nebel (Foto: (c) Foto Homberger Arosa). 
(auch wenn die tarifarische Honorierung dafür gering ist). Denn man will ja den Patienten nicht als den mit der Schulterluxation oder mit der akuten Appendicits (der natürlich ins Spital im Tal gehört) etikettieren, man möchte den Menschen vor sich sehen, ob er nun ein Geschäftsführer einer führenden Gastronomiekette, ein ETH-Professor aus Lausanne, eine erfolgreiche junge Buchautorin aus Paris oder ein Mädchen aus der 2. Klasse ist.

\section{Und dann will man kompetent behandelt werden, und das rund um die Uhr.}

Das ist doch wie in einer andern Praxis, mag man hier einwenden. Nein, nicht ganz, denn die geografische Lage macht die Praxis in den Bergen etwas spezieller. Das medizinische Spektrum ist deshalb sehr breit und der Anspruch der Patienten zum Teil sehr hoch, denn Krankheit oder Verletzung haben keinen Platz in den Ferien. Und dann will man kompetent behandelt werden, und das nicht nur von $8 \mathrm{Uhr}$ morgens bis $6 \mathrm{Uhr}$ abends, nein, rund um die Uhr, denn bekanntlich richtet sich der Notfall (oder das, was der Patient als solchen anschaut) nicht nach der Uhrzeit. In der Saison ist eine Ambulanz vor Ort, und die medizinische Praxisasssistentin (MPA) hilft mit bis 22 Uhr. In der übrigen Jahreszeit werden diese Dienstleistungen aber heruntergefahren, weil es nicht rentiert (!), und die Tal-/Bergbewohner und ihr Arzt sind wieder so ziemlich alleine. Mit Zusammenlegen von medizinischen und sozialen Einrichtungen wird versucht $\mathrm{zu}$ sparen. Und plötzlich dauert es länger, bis man am Talboden angekommen ist...

Oben arbeitet derweil eine Praxiscrew, zusammengesetzt aus Ein-heimischen und Nicht-heimischen. Und sie trotzen nicht nur dem Unbill des Wetters: Der Lohn unten, die Arbeit ist sehr vielschichtig und lang, man hat häufig Dienst und das soziale und kulturelle Angebot ist halt weniger üppig, zumindest in der Zwischensaison. Aber sie tun es trotzdem, die medizinischen Praxisassistentinnen, die man(n) nicht genug schätzen kann, und die Ärztinnen und Ärzte, die plötzlich kein grosses Spital mit all den topmodernen Abklärungsund Behandlungsmöglichkeiten im Rücken wissen und sich deshalb wieder mit der medizinischen Basis begnügen (müssen).

Sie tun es trotzdem. Weil - die Arbeit eben äusserst spannend ist, abwechslungsreicher und fordernder, weil das Leben ausserhalb des Grossstadtsmogs "natürlicher» ist, es weniger nieselnden, trüben Nebel gibt, viel Natur ermöglicht - es gibt sie noch, weil es anders ist. Für jetzt oder für länger. Weil das Leben trotz allem gelassener fliesst. "When you hit it real hard, and you feel the pain, you gotta reach inside. Find sunshine and rain" singt der Berner Blueser, Philipp Fankhauser [2]. Und wenn sich dann eben Genuss einstellt, just «let it roll» [3].

\section{Wer da oben arbeitet, dem gehört Respekt und Wertschätzung!}

Und darum - wer da oben arbeitet, dem gehört Respekt und Wertschätzung! Und dies nicht als Lippenbekenntnis! Eigentlich tragisch, wie das immer wieder vergessen wird.

PS: Für Politiker, Gesundheitspolitiker, Verwaltungsräte und Tourismusverantwortliche kann Geschriebenes auch konkret «ausgedeutscht» werden.

Literatur

1 D. Heim. Le médecin de campagne - der Film. SAEZ 2016:97(36):1249-51.

2 Philipp Fankhauser. Let life flow (Funk House Blues Production, 2019).

3 Little Feat. Let it roll (Warner Bros Records 1988). 